\title{
A Validation Study of Merging and Spacing Techniques in a NAS-Wide Simulation
}

\author{
Patricia Glaab, ASAB, NASA Langley Research Center, Hampton, VA
}

\begin{abstract}
In November 2010, Intelligent Automation, Inc. (IAI) delivered a software tool to NASA Langley that models Merging and Spacing (M\&S) for arrivals and departures in the Airspace Concepts Evaluation System (ACES) NAS-wide simulation. This delivery allows researchers to use ACES for system-level studies of the complex terminal airspace. As a precursor to use of the tool for research, the software was evaluated against current day arrivals in the Atlanta TRACON using Atlanta's Hartsfield-Jackson International Airport (KATL) arrival schedules, Standard Terminal Arrival Routes (STARs), and traffic flow management (TFM) techniques typical for Atlanta. Results of this validation effort are presented describing data sets, traffic flow assumptions and techniques, and arrival rate comparisons between reported landings at Atlanta versus simulated arrivals using the same traffic sets in ACES equipped with M\&S. Since emphasis for intended research is on arrival capacity, this was also the focus of the validation.
\end{abstract}

Before testing began, the simulated system was expected to demonstrate superior capacity over current day Atlanta by managing spacing intervals efficiently and exactly. Initial results, instead, showed the simulation's modeled capacity to be far short of what human controllers currently achieve, despite the efficiently managed spacing. Investigation into the cause of the shortfall revealed aspects of systems-level flow and control techniques that are critical to achieving sustained high capacity in the face of varying traffic loads and type mixes. This new understanding, once applied to the current day validation model, allowed a match of Atlanta's arrival capacity as well as a better understanding of how modern airports are limited by current day route models.

Following this validation effort, a sensitivity study was conducted to measure the impact of variations in system parameters on the Atlanta airport arrival capacity.

\section{Motivation}

As systems-level simulations mature and evolve, the terminal airspace becomes the subject of an increasing amount of research because of the key role this area plays in system-wide capacity and delay. Recent innovation and development of trajectory modeling and traffic flow in the TRACON for NASwide simulations now makes it possible to study this airspace with respect to large volumes of traffic over the course of full days. The inter-dependence of airborne spacing, runway spacing, aircraft performance, and traffic mix create a complex system that is sometimes difficult to predict and can yield non-intuitive results when modified.

Modern improvements in aircraft navigation capabilities offer the potential for airports to achieve improvement in arrival and departure capacity. By simply reducing the required distances between aircraft, airborne capacity can be increased without changing current day routing in any other way. However, significantly larger gains are possible if the routes themselves are designed to take better advantage of improved navigation capabilities. By simulating and studying possible future route concepts, based on techniques validated against current-day traffic flow, identification of designs that offer the greatest capacity and delay improvement in 
the time frame in which new technologies are expected to be available are possible.

In November 2010, Intelligent Automation, Inc. (IAI) delivered a software tool to NASA Langley that models Merging and Spacing (M\&S) for arrivals and departures in the Airspace Concepts Evaluation System (ACES) NAS-wide simulation. This delivery allows researchers to use ACES for system-level studies of the complex terminal airspace. As a precursor to use of the tool for research, the software was evaluated against current day arrivals in the Atlanta TRACON using Atlanta's Hartsfield-Jackson International Airport (KATL) arrival schedules, Standard Terminal Arrival Routes (STARs), and traffic flow management (TFM) techniques typical for Atlanta.

While matching a single airport under a single set of operating conditions is not a blanket validation of all scenarios, it is a necessary first step to a fuller understanding of the capabilities and limitations of the tool as well as to better understanding the nuances of capturing real-world traffic flow with a systemslevel simulation tool.

Before testing began, the simulated system was expected to demonstrate superior capacity over current day Atlanta by managing spacing intervals efficiently and exactly. Initial results, instead, showed the simulation's modeled capacity to be far short of what human controllers currently achieve, despite the efficient managing of spacing. Determining the cause of the shortfall revealed aspects of systems-level flow and control techniques that are critical to achieving sustained high capacity in the face of varying traffic loads and type mixes. This new understanding, once applied to the current day validation model, allowed a match of Atlanta's capacity as well as a better understanding of how modern airports are limited by current day route models.

Once current day arrival capacity was achieved, route and spacing parameters were varied to determine the effect on the overall system. Lessons learned are supporting research for future concept routes, which is the intended follow-on study to this work.

\section{Simulation Summary}

This validation study was conducted between October 2010 and February 2011 and used the ACES NAS-wide simulation configured with the M\&S plug-in [1]. The M\&S tool performs functionality in the simulation that would typically be provided by TRACON flow controllers in real world air traffic operations. As flights approach real Atlanta, they are handed off to the TRACON control from the Center, and TRACON controllers direct the flights to the appropriate STAR arrival. In the simulation, this function is performed by the $M \& S$ tool [2] as simulated flights enter the region of control (ROC) defined for the airport. For this simulation study, the ROC was defined as a circle with a 200 nautical mile radius around the aircraft center. Arriving flights are handled on a "first-come-first-served" basis.

The M\&S tool imposes time separation constraints on managed aircraft, based on FAA spacing requirements [3] for miles-in-trail (MIT) when aircraft enter the ROC. Time spacing is then maintained between aircraft as flights slow and descend toward landing. This time-based approach to separation is consistent with airborne separation assurance techniques currently being researched by NASA which target wake dispersion time requirements rather than fixed distances.

Routes were configured to model the ERLIN, FLCON, CANUK, and HONIES arrivals into Atlanta Airport. The behavior of the flights from the time they entered the Region of Control (ROC) of the M\&S system (200 nautical mile radius around the airport center) until they touched down on the runway was the primary focus of this study. The core ACES simulation has the ability to manage flow at several stages of the flight. For this study, all of the core ACES simulation delay and scheduling capabilities were disabled, allowing $M \& S$ exclusive control over the timing of route navigation. 
The 2006 Baseline Day dataset was the primary traffic data used, along with a compiled dataset created for the study from operations logged by Atlanta on October 25, 2010. The M\&S tool was configured to contain routes and vectors to match those of Atlanta, and the arrival performance was tested using the 2 datasets. During the testing phase, guidance was sought from the Atlanta TRACON traffic flow managers to verify assumptions made in the setup and to provide guidance on flow techniques.

Time required to conduct simulation runs was a consideration. The datasets and runway configurations used for the sensitivity study often intentionally overloaded the simulated TRACON which significantly extended the time required for a simulation run because of the additional trial planning required. Ideally, no more than the minimum path stretch necessary would be added to flights to maximize the throughput of the system. However, the smaller the delay interval, the more attempts must be made before achieving the full delay required for any given flight. To offset the effect of a small delay granularity (10 seconds), the flight data set size was reduced by eliminating all flights not bound for Atlanta. This allowed even the most heavily loaded flight sets to complete their simulation runs in 10 hours or less. Interactions with non-Atlanta-bound flights were obviously lost, but were not integral to the results of this study.

The KTG option was used for trajectory modeling in ACES, and provided geometric paths in 4-dimensional space all the way to the runways. The M\&S tool coordinated crossings of predicted trajectories over arrival route waypoints appropriately for the aircraft type and waypoint constraints, and imposed delay maneuvers when necessary to prevent spacing violations. The shortest path was investigated first, but alternate paths were used if the imposed delays became large enough to warrant them. Prior to delivery of the M\&S software, verification of the tool was conducted by the Intelligent Automation, Inc. In that phase, testing was done to confirm that algorithms governing spacing requirements, route and runway assignments, and trajectory selection were properly implemented and followed.

\section{Selection of Atlanta Airport}

Atlanta's Hartsfield-Jackson International Airport, known locally as Atlanta Airport (Figure 1), is one of the world's busiest airports with respect to passenger traffic and number of landings and takeoffs. The airport is the primary hub of AirTran Airways, Delta Air Lines, and Atlantic Southeast Airlines and handles almost one million operations annually. Because of its volume, Atlanta is the popular subject of much research and attention seeking to understand its challenges and triumphs. Availability of prior research data assisted startup research for this effort.

Since Atlanta Airport is located in the central eastern part of the US, it receives arrivals from all directions. Additionally, it is located in a region with relatively few airspace constrictors (like mountains or Special Use Airspace). This results in a very diverse collection of flown track data into the Atlanta TRACON and suggests that Atlanta could later be more easily generalized for application to concept routing.

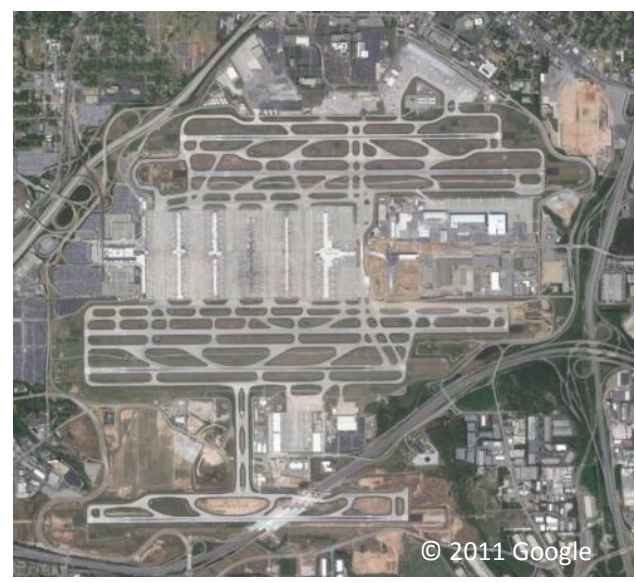

Figure 1. Atlanta's Hartsfield-Jackson Airport [4]

Atlanta was also an attractive choice for this validation study because the efficient design of the airport reduces the complexity of modeling it. Atlanta has 5 runways available, allowing it to 
dedicate specific runways for either arrival or departure. This eliminates the added burden of modeling interleaved arrival and departure traffic to a single runway. Arrival runways are spaced far enough apart to allow them to operate autonomously without hindrance of closely spaced parallel runway rules. Taxiing aircraft generally do not cross active runways. While the M\&S tool could model these complexities, post-analysis of runs would be significantly more time-consuming. Finally, the same STAR arrivals are used regardless of the number of runways in use or the landing direction (by specifying final STAR legs by runway). This allowed flexibility in runway configuration with minimal setup work.

\section{Configuration of Routes}

STAR arrival procedure details were obtained from AirNav [5]. Atlanta Airport has eleven Standard Terminal Arrival Routes (STARs) available, but uses four predominantly - FLCON, ERLIN, HONIE, and CANUK [7]. ERLIN is used for arrivals from the northwest, FLCON for arrivals from the northeast, CANUK for arrivals from the southeast, and HONIE for arrivals from the southwest. (See Appendix A for copies of procedure plates obtained from AirNav.com[5].)

The STAR definitions, however, do not contain critical information for traffic flow between the end of the STARs and designated runways. For this phase of the arrival, the STAR merely instructs pilots to "expect vectors". In practice, controllers issue vectoring instructions to the aircraft to perform efficient merging with adequate spacing of flights to the final approach fixes. Typical vectoring patterns were obtained through observation of the Airport Tracking display on the FlightAware.com website (Figure 2), and were incorporated into the configured routes for the simulation runs (Figure 3). To verify proper assignment of route waypoints, the simulated runs were compared to the observed real-time traffic using the ACES Viewer visualization tool [6].
Atlanta transitions several times a day between single, dual, and triple runway arrivals to accommodate arriving flow. Triple runway operations are only used when necessary to accommodate arrival volume and minimize taxi distance required by aircraft to reach the terminal after landing. Atlanta operates with westward runway flow $70 \%$ of the time [7]. This westward runway configuration has the added benefit of allowing the most crowded northeast corridor traffic the more efficient "short side" of the arrival pattern with a straighter route to final approach. Traffic arriving from the west, on the "long side", must travel a downwind and base leg before entering final.

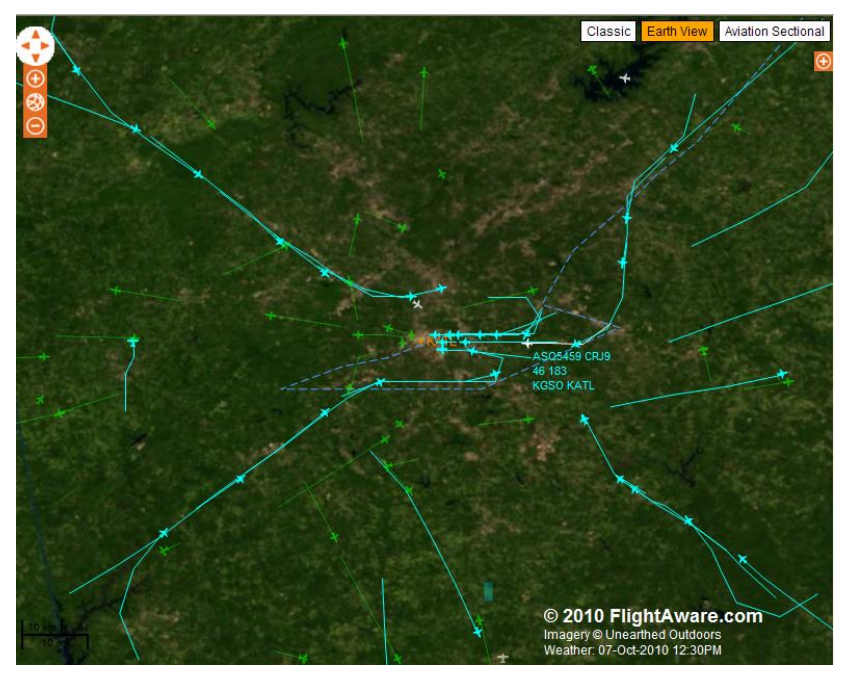

Figure 2. Atlanta, 3-runway operation, westward flow

A limitation of the current version of the M\&S tool is its inability to change the available routes or runways mid-run to better simulate real-world dynamic airport configuration by ATC. The tool was configured to use 3 runways for the entire simulation run. Since the maximum capacity values for realworld Atlanta occurred during 3 runway operation, this allowed the simulated and real-world systems to match for the periods of interest for the full day traffic testing. (During the follow-on sensitivity study, single runway operation was sometimes used and is discussed later in that section.) 


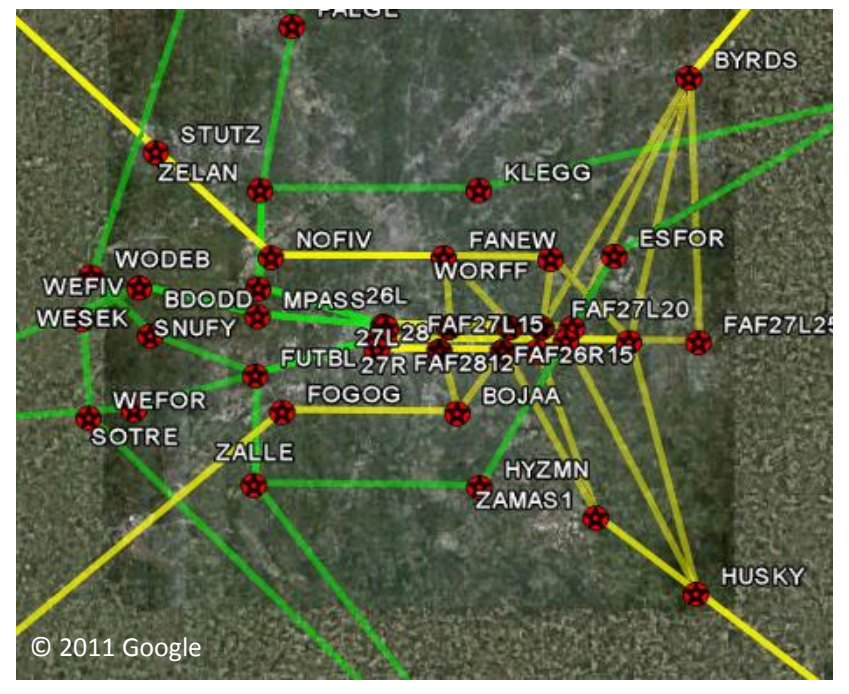

Figure 3. Configured arrival and departure routes [4]

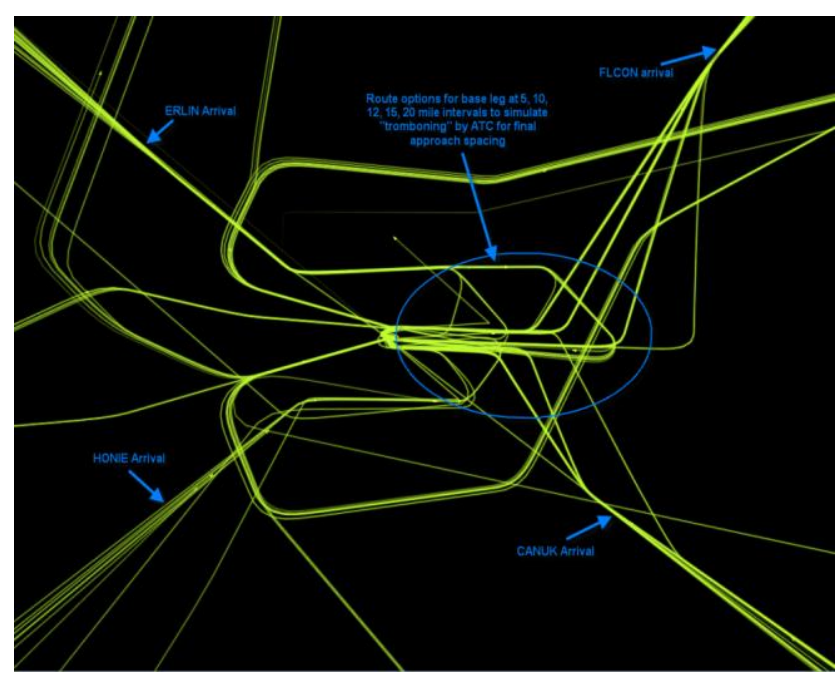

Figure 4. Simulated arrival and departure tracks [6]

With few exceptions during 3 runway operations, the ERLIN and the FLCON arrivals use runways 26R and $27 \mathrm{~L}$. The CANUK arrivals most often use 28 , but can be vectored to $27 \mathrm{~L}$ to fill gaps in the flow. HONIE arrivals travel south of the airport and land on 28 , or are directed to the north of the airport and merge with the ERLIN arrivals on the downwind leg.

To complete the route configuration, legs were added to model vectoring from downwind to final for the ERLIN and HONIE arrivals from the west and to vector from the end of the FLCON and CANUK STAR to final. As a final check, flown tracks from simulated routes were inspected and compared back to the original Atlanta traffic patterns.

\section{Establishing a Baseline}

Flights are fed into the Atlanta TRACON by the Center at the acceptance rate set by the TRACON. The TRACON Flow Manager sets the value based on current weather conditions and with regard to any current runway issues (for example, an ILS may be temporarily out of service). Atlanta advertises an arrival capacity of 126 arrivals per hour to the Center during peak operations in clear conditions, with actual arrival volume generally measuring 100-115 aircraft/hour. Before running the full 2006 Baseline Day of traffic with the simulation, the first tests used a small subset for the midday, high volume period. However, in the initial testing of the simulated route configuration for Atlanta, arrival capacity peaked at 85 aircraft per hour when measured over 60 minute intervals for the 2006 Baseline Day dataset-a substantial shortfall (Figure 5).

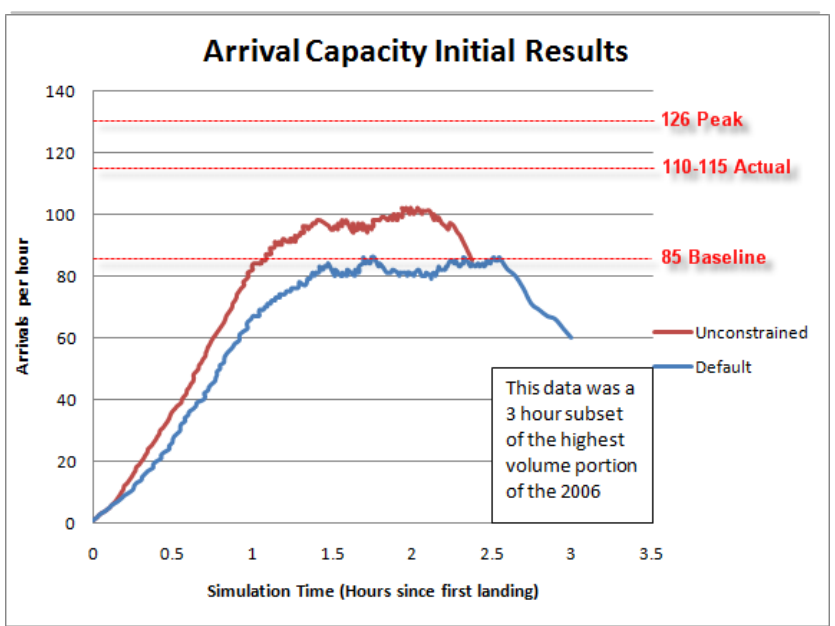

Figure 5. Initial Capacity of 85 flights Per Hour

The possibility that differences in the computation of arrival rates were giving the appearance of a shortfall had to be eliminated. In a fluctuating volume, larger sampling intervals can masquerade as smaller overall rates because of the effect of averaging. Before discounting the use of different sampling intervals, an assessment was made 
of the interval being used for reporting actual Atlanta arrival rates. FlightAware [8], which publishes arrival and departure data in real-time, was used as the data source for validating the time interval. FlightAware arrival data was gathered for a period spanning 24 hours, sorted, and analyzed. Various sampling periods were tested to determine which one most closely matched the graphical data for the same time frame (Figure 6). Each line in Figure 6 used the exact same data, but applied different sampling intervals to calculate arrival rate by normalizing to an hour:

$$
\mathrm{A}=\mathrm{N} * 60 / \mathrm{t}
$$

Where:

$$
\begin{aligned}
& \mathrm{A}=\text { Arrival Rate (aircraft/hour) } \\
& \mathrm{N}=\text { Number of arrivals in sample } \\
& \mathrm{t}=\text { Number of seconds in sample }
\end{aligned}
$$

For example, 40 arrivals counted in a 30 minute sample would yield an arrival rate of 80 per hour.

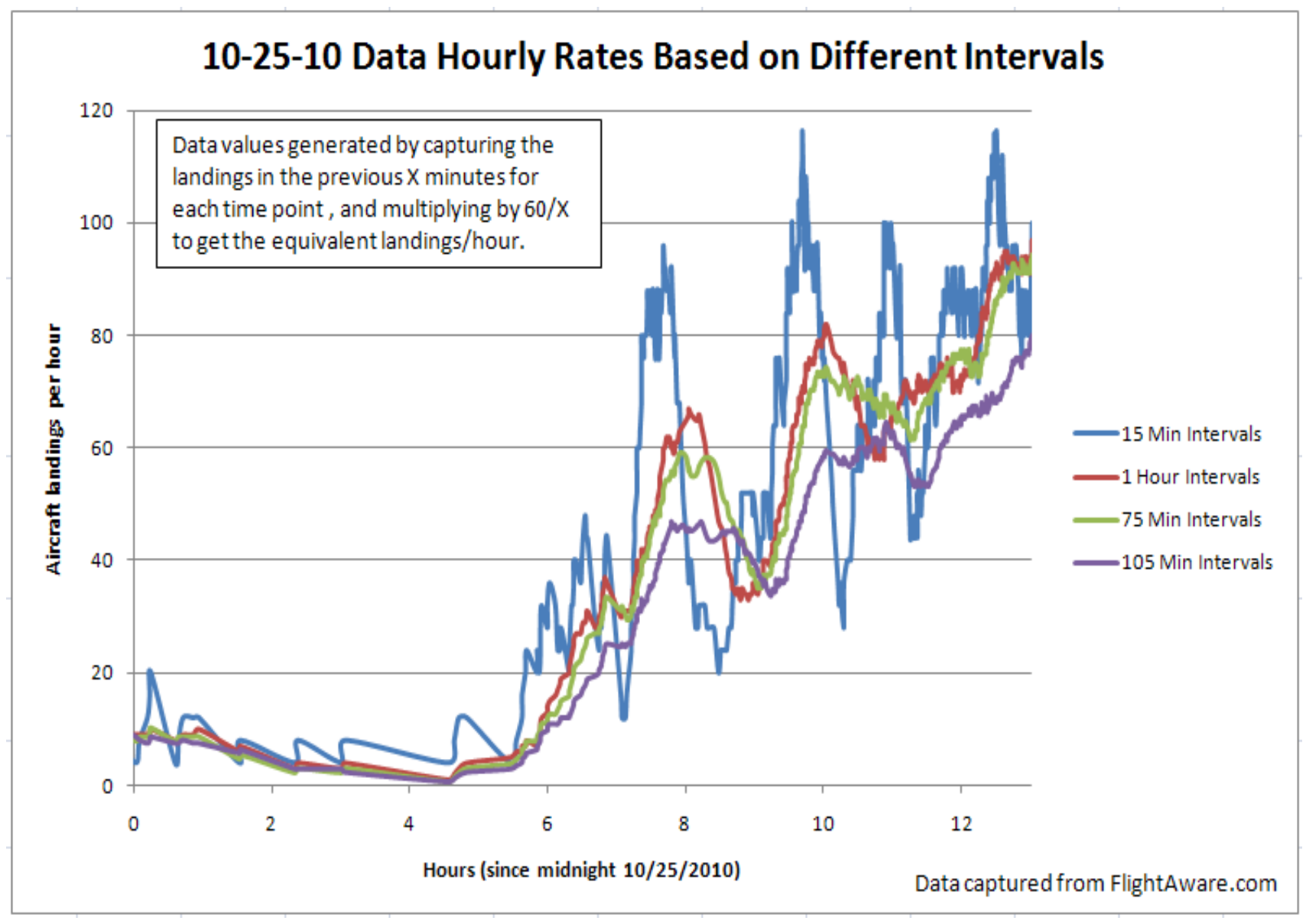

Figure 6. Effect of interval selection on amplitude 


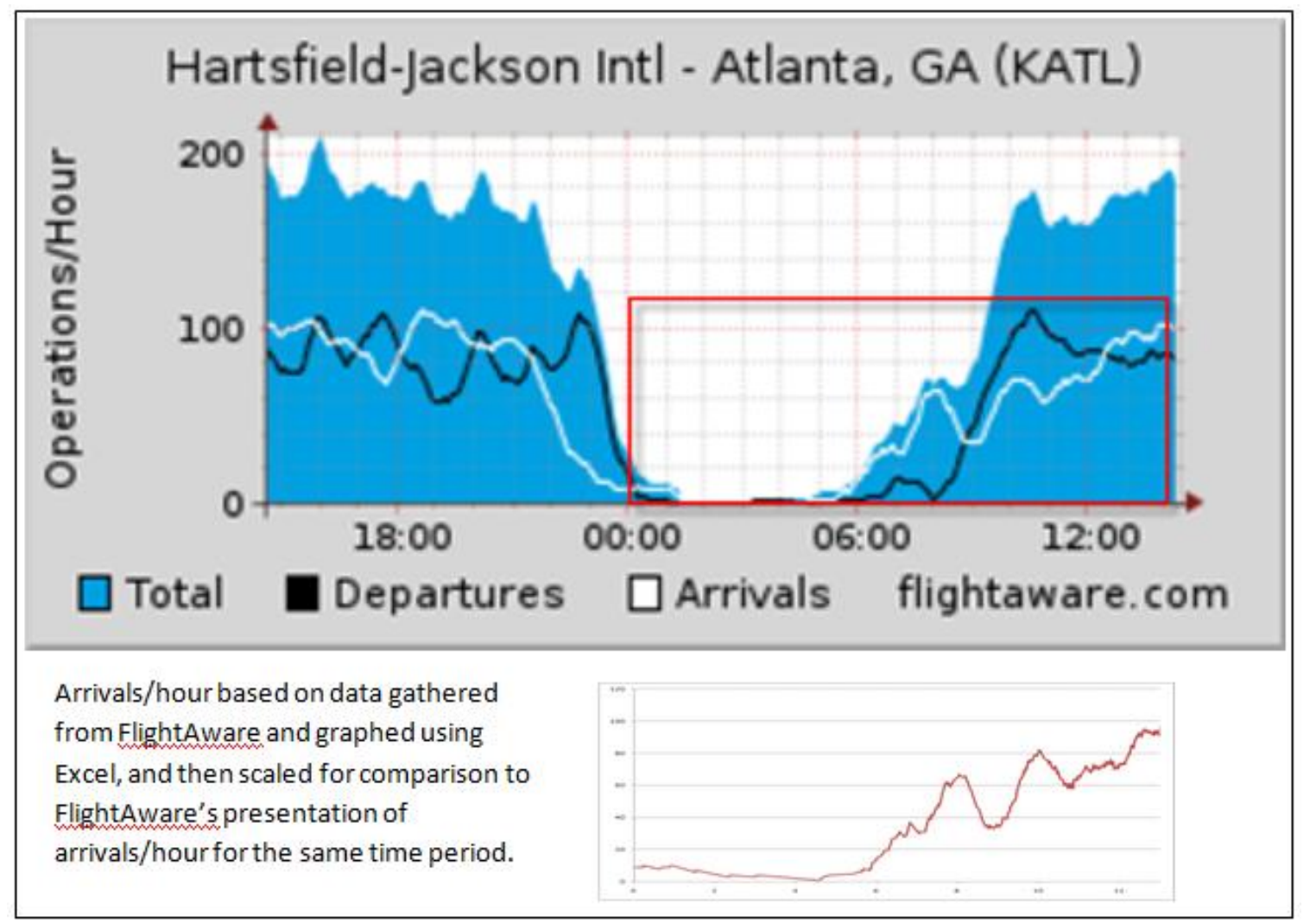

Figure 7. FlightAware graphical arrival rate data compared to tabulated data for the same time period

The tallied 60-minute data was the nearest match, as originally suspected (Figure 7). Some small differences exist between the tallied landings and the FlightAware graphical data. These are believed to be partly due to the timing of the reported data, which is sometimes delayed by more than an hour after the actual landing. The tallied data is pre-sorted to avoid missing late entries, but the FlightAware data is not retroactively corrected. Also, a small number of flights are reported twice. These were removed from the captured data, but were (presumably) rolled into the FlightAware graphical data as reported.

The confirmation of the 60-minute interval unfortunately also confirmed the initial capacity shortfall of the arrival routes as modeled, and prompted closer inspection of route nuances and further discussion with Atlanta TRACON personnel to improve the configured route model.

\section{Resolving the Initial Shortfall for the Simulated Airport}

\section{Assessment of Traffic Volume Provided by Data Set}

An inspection of the traffic data set was made to confirm that the 2006 Baseline Day data contained enough flights into Atlanta to produce the required $110-115$ aircraft/hour volume. To verify adequate traffic in the data set, a run was made with the system configured with minimal constraints. For a typical run, flights are initiated in the simulation at a time specified in the traffic configuration file, travel to their destination at a rate determined by the that aircraft's performance capabilities and selected flight plan, and are then delayed in the terminal airspace by the Merging and Spacing algorithms as necessary to meet FAA wake and runway spacing requirements. For the minimal constraint test, however, all imposed wake and runway spacing 
was disabled. Traffic was allowed to flow at a rate determined only by performance and departure time. In this case, the traffic volume was measured to be 123 arrivals per hour (Figure 8), which was well above the 85 aircraft per hour seen with the standard spacing enforced. This verified that the volume of traffic in the data set was adequate for this test.

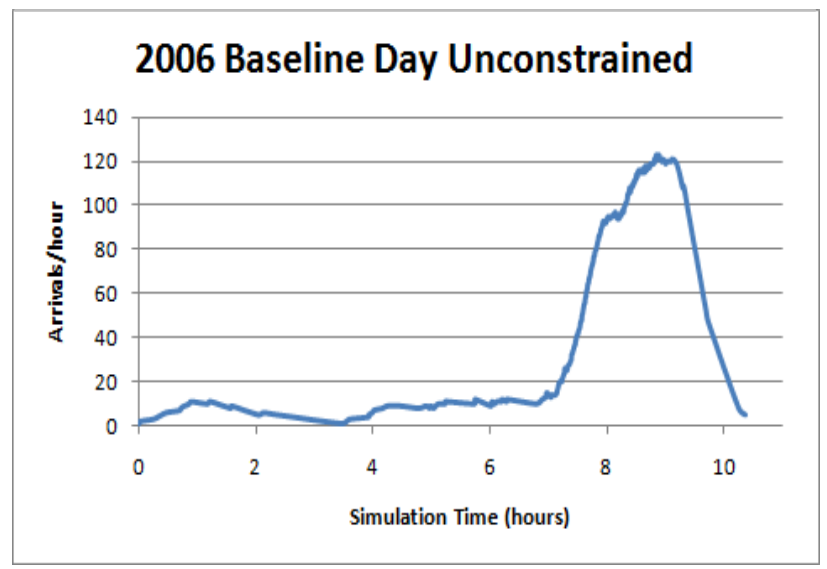

Figure 8. Unconstrained 2006 Baseline Day Arrivals

\section{Traffic Mix and Origin Airport Characteristics}

The possibility that differences in traffic type characteristics between current day and 2006 (the source of the Baseline Day data) were causing flow discrepancies had to be eliminated as a reason for the shortfall. For example, 2006 Atlanta traffic might result in significantly higher mismatches in leader/follower pairs on an arrival route. A second traffic data set was created to assess whether the current day traffic mix is consistent with the mix of traffic in the 2006 Baseline Day. The new data set was created using the traffic profile previously captured for the sampling interval test. For each flight in that set, departure airport, departure time, arrival airport, arrival time, and aircraft type were available. Cruise altitude, airspeed, and track data were borrowed from the 2006 Baseline Day by type for a particular departure airport. The FlightAware sample day was October 25, 2010, a clear weather day for much of the country. This traffic set was run with unconstrained flow to baseline it, and was then run through $M \& S$ with standard FAA spacing enforced (Figure 9). If the traffic type mix were a significant effect, the October 2010 data set would have resulted in a significantly different maximum capacity than the 2006 data set. However, the traffic throughput again peaked at 85 aircraft per hour with a dataset capable of supplying more volume (95 flights per hour).

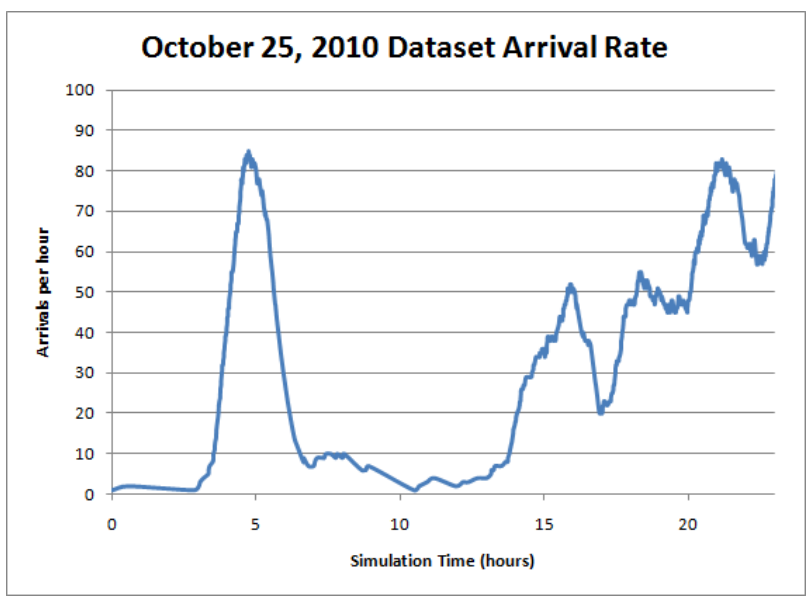

Figure 9. Initial arrival rate with 10/25/2010 dataset

\section{Path Stretch Delay Granularity}

A slight improvement with the simulated arrival capacity was seen with the November (final) delivery of the M\&S tool. The improvement was due to the use of path stretch maneuvers, rather than holding patterns, to delay for spacing. The path stretch provided a 10 second delay granularity compared to the previous 4 minute granularity of a holding pattern. The improvement in delay granularity resulted in an arrival capacity increase from 85 to 90 flights per hour.

\section{Addition of Tromboning}

Another improvement to capacity was achieved when route sections were added to mimic extensions on the downwind leg, often called 
"tromboning" (because the back and forth movement of the flown tracks with time is reminiscent of the motion of the slide arm of a trombone). In the real-world, these patterns are the result of vectoring commands from controllers and are referred to, but not detailed, in published STAR arrival procedures. The originally configured route depicted the arrivals only as detailed by the STAR procedure (Figure 10). When the configured routes were expanded to include modeling of tromboning (Figure 11), a significant improvement in merging flexibility was gained for the lower portion of the route, and arrival capacity increased from 90 to 95 aircraft per hour for simulation (Figure 12).

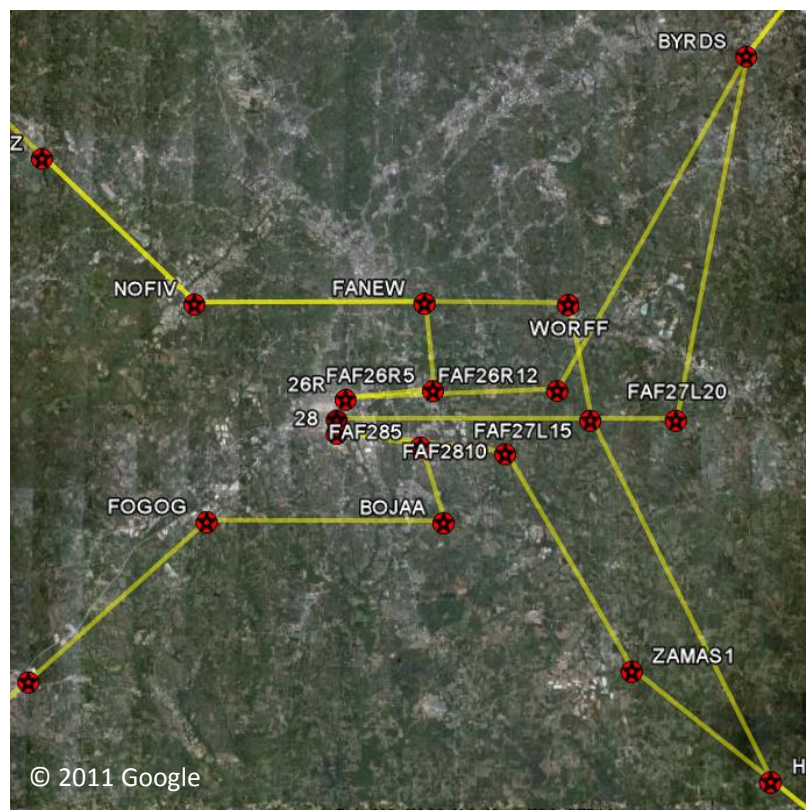

Figure 10. Original Route Configuration [4]

The selection of the merge points of the trombone options to final approach was made after careful examination and measurement of traffic using the FlightAware.com Flight Tracker display. FlightAware observations also resulted in the selection of the number of optional vectors from any given route to final for any given runway. Some consideration had to given to the $M \& S$ tool capabilities since additions and restrictions to route options cannot yet be made during mid-run. For example, approaching traffic from the northwest to runway $27 \mathrm{~L}$ is routed around traffic from the same route to 26R. In real-world Atlanta, the downwind leg length would be shortened if the traffic to $26 \mathrm{R}$ were using a shorter base leg, but this was not possible for the simulation, which was configured to always use a 20 mile base leg for the northwest to runway $27 \mathrm{~L}$ connection.

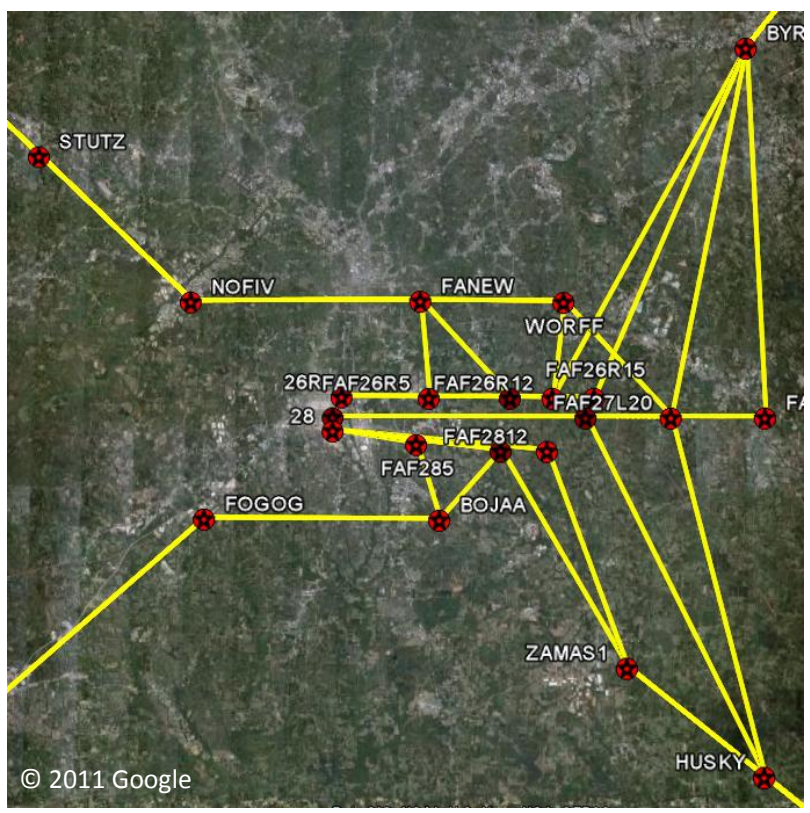

Figure 11. Routes With Tromboning [4]

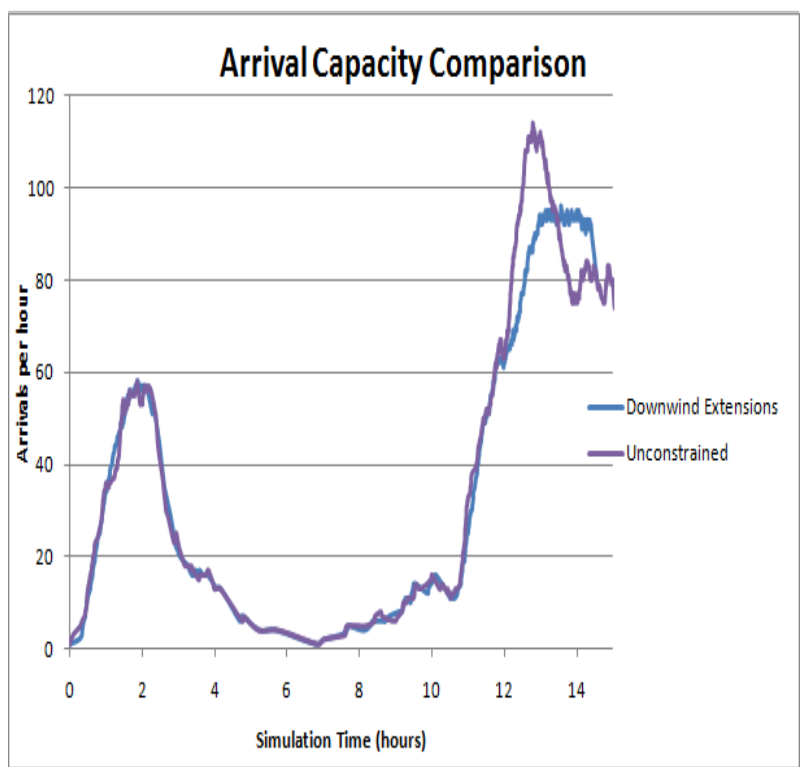

Figure 12 - Arrival Capacity With Tromboning 


\section{Hold-Speed Instructions}

A comparison of touchdown intervals was made for simulated versus real-world arrivals. Real-world Atlanta consistently touches down aircraft with near minimal spacing during high volume operations. Simulated Atlanta, however, showed a majority of intervals that were significantly larger than the minimum, yet too small to fit a merging aircraft. This resulted directly in wasted capacity for the simulated system.

Close inspection of short-term bursts of simulated arrivals revealed significantly higher efficiency for aircraft instructed (by the $M \& S$ system) to follow a lead aircraft along STAR legs. However, observation of merging patterns for realworld Atlanta using FlightAware indicated that increasing the number of arrivals using following instructions before final would not be consistent with observed traffic flow. Significant merging was performed late in the stream, and would preclude higher use of following down the outer portion of the STAR than was already occurring with the tool.

The necessary final improvement to achieve an arrival capacity match was made by maintaining the prior improvements (tromboning vectors and improved arrival route-to-runway options) and adding trajectory speed adjustments. Specifically, aircraft were instructed to select up to a $10 \%$ airspeed increase over their default (high efficiency) trajectory when the slot was available when selecting an arrival schedule. This caused flights to close the gap on the aircraft ahead of them. Though the consequence of this is reduced fuel efficiency as aircraft are steered from their optimal speed, it compacts the flow forward and removes wasted capacity between aircraft allowing the combined, reclaimed space to become available for following aircraft.

In the simulated model, this was achieved by directing the tool to select the "Earliest" arrival time to the next waypoint, rather than the more fuel- efficient "Default" trajectory. The M\&S tool does not allow application of this speed change opportunistically, however, as is done by actual controllers. For fuel efficiency, the Default (rather than the Earliest) trajectory is more optimal. However, the priority in this case was capacity. With the addition of the trajectory speed increase, the arrival rate finally matched the 115 arrivals per hour rate for high volume operations seen at Atlanta (Figure 13).

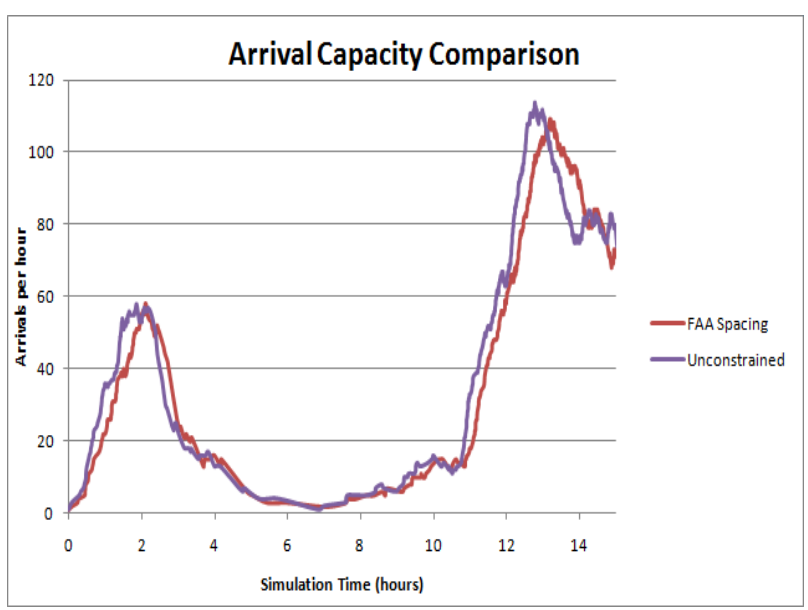

Figure 13. Final Capacity Match, 2006 Baseline Day

A close inspection of the arrivals per hour while using Default trajectories versus Earliest trajectories demonstrates how the capacity gain is achieved. With the same traffic data set, the Earliest trajectory arrival rate leads that of the Default trajectory (Figure 14).

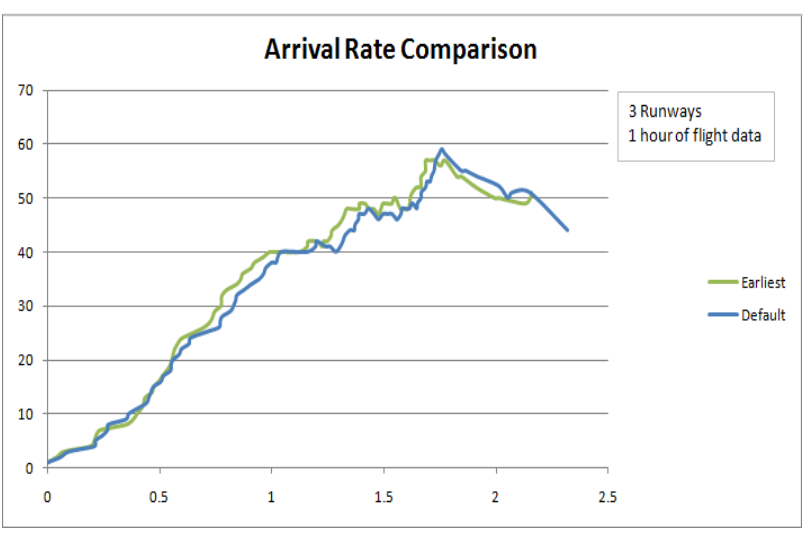

Figure 14. Default vs. Earliest Trajectory Schedulers 
Each delaying increment represents 10 seconds of delay. By compacting traffic forward to remove wasted capacity between flights, the total system delay is reduced from 102 delaying increments (17 minutes) to 68 delaying increments (11.3 minutes) for the same traffic set (Figure 15). As a visualization, picture a loosely strung set of beads on a wire being pushed together to fit more beads on the end.

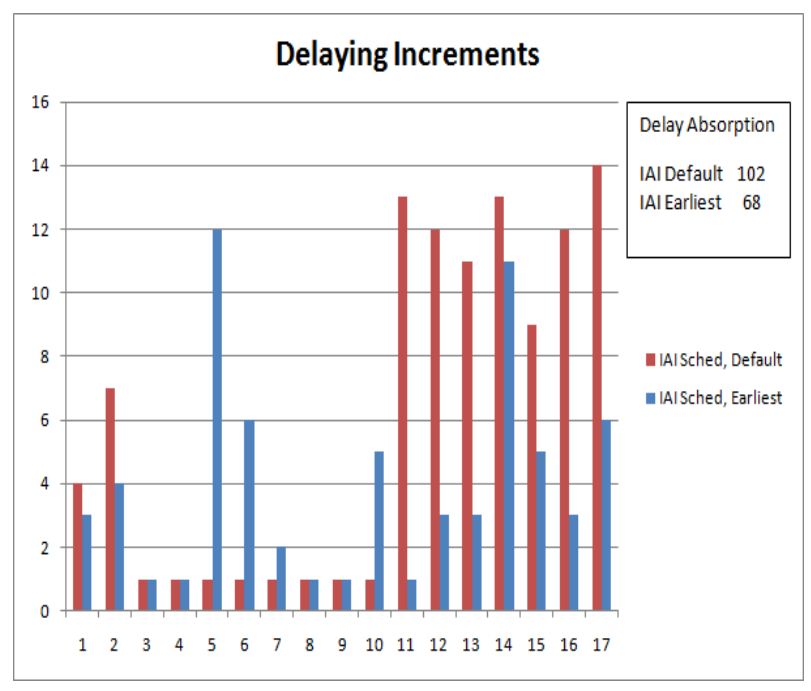

Figure 15. Total Delay, Default vs. Earliest Trajectories

Following these findings, the validity of this technique was discussed with the supervisor at the Atlanta TRACON. Specifically, he was asked if increasing the speed of arriving aircraft to improve capacity would be used in real world operations. The reply was that this technique was actually the first thing they did when anticipating a period of high volume. In practice, aircraft are instructed to hold speed when handed off from the Center Controller, rather than allowing them to slow to the default speed for the STAR entry.

\section{Theoretical Maximum Capacity}

During conversations with Atlanta flow controllers, the comment was made that if runway occupancy spacing were the only restriction (i.e., no in-air wake spacing was required), Atlanta could advertise an arrival rate of 150 aircraft per hour to the Center, but they were limited to only 126 arrivals per hour because of wake spacing.

The M\&S software models wake spacing and runway occupancy spacing separately, and allows either or both to be disabled. This anecdotal case was tested with the M\&S system by disabling wake spacing requirements while maintaining those for runway occupancy spacing. Supplying adequate traffic volume was an issue. Since traffic data is based on flown tracks which obeyed real-world arrival rates restrictions, no dataset was available to supply 150 aircraft per hour to Atlanta. To approximate the condition, the simulation was configured with a third of the runways (1 runway rather than 3) and used a portion of the 2006 Baseline Day dataset that supplied a little more than a third of the theoretical potential capacity (Figure 16).

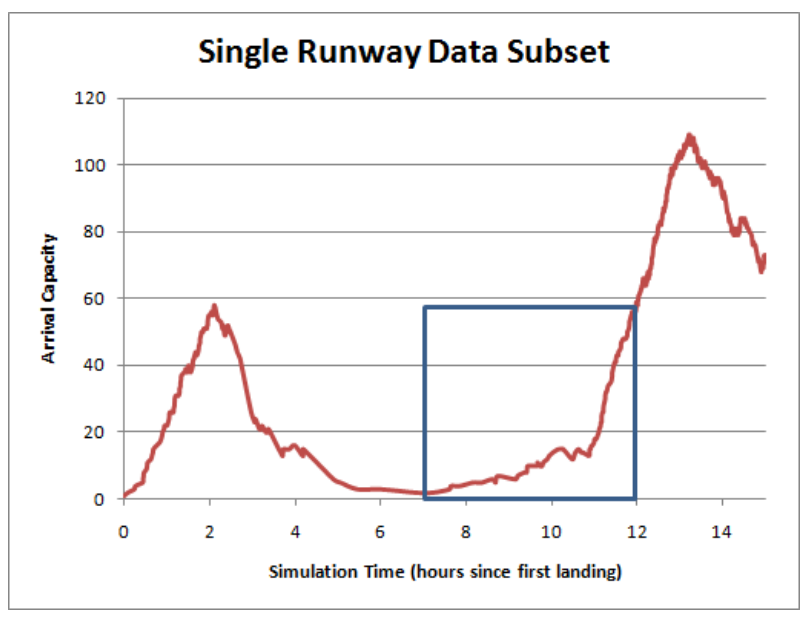

Figure 16. 2006 Baseline Day Data Subset

FlightAware was again enlisted to properly configure routes and runways, and to select merge points for vectoring (tromboning) from the ends of the STAR arrivals to the runways (Figure 17).

For a perfect match, the scaled simulation data should be limited to 42 arrivals per hour (126/3) for 
the case using standard spacing, and 50 arrivals per hour (150/3) for the case with wake spacing disabled.

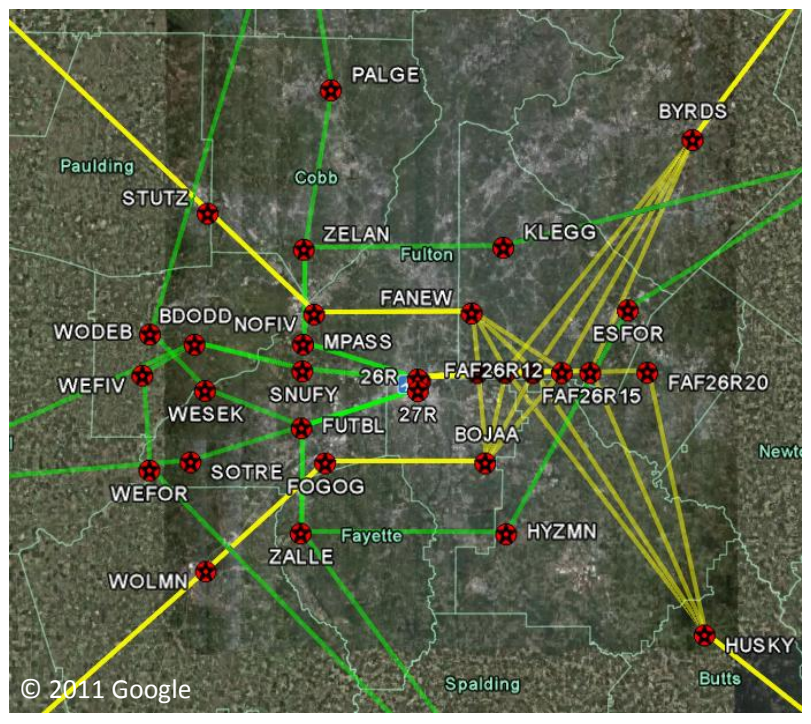

Figure 17. Single Runway Capacity Configuration

The simulated runs demonstrated a very close match with the standard spacing run peaking out at 42 arrivals per hour, and the case with wake spacing disabled peaking at 52 arrivals per hour (Figure 18).

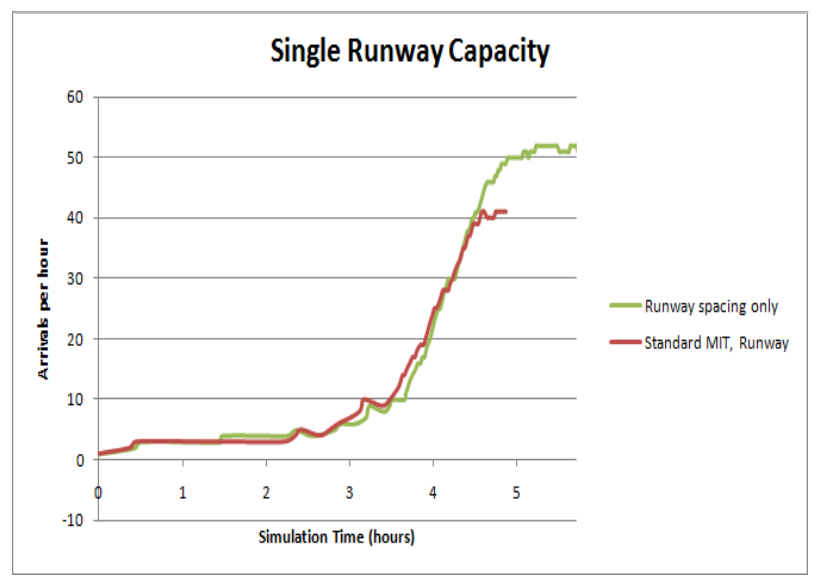

Figure 18. Capacity Test of Anecdotal Case

\section{Validation Study Conclusions}

The simulated Atlanta demonstrated a good match to real Atlanta when several critical flow techniques were captured for the modeled routes. The first was proper connection of arrival routes to runways, regarding both the number of runways connected for an individual route and regarding adequate diversity in vectors from the bottom of the STAR arrival to final approach. Reducing either the number of runway connections or the vectoring options reduced the overall capacity of the system by about $12 \%$ for the datasets tested ( 85 versus 95 aircraft per hour).

The other critical technique was maintaining higher arrival speeds for the aircraft entering the terminal airspace during high volume operations to push them down the routes and compact the flow. This change resulted in more than a $15 \%$ increase (110 versus 95 aircraft per hour) in arrival capacity. Both the application of vectors and the trajectory speed adjustments used for the simulation route models are consistent with techniques used at the Atlanta TRACON.

\section{Sensitivity Comparisons}

Once the overall arrival capacity was matched for Atlanta, experimentation was done with imposed spacing intervals to quantify their impact on arrival capacity for this simulation and this dataset.

In the first set of runs, the runway occupancy spacing was disabled, while the wake spacing value was varied from 0 (the unconstrained case) to $100 \%$ of standard FAA values (order of arrivals was enforced). Standard FAA wake spacing requirements vary depending on size category of the leader and follower. For this test, a consistent percentage was applied for all leader/follower combinations for a given run.

In the second set of runs, the wake spacing was disabled, while the runway occupancy time was varied from 0 (serving as the unconstrained case) to 
72 seconds (the average time between touchdowns seen in the October 25, 2010 dataset).

As expected, the overall capacity decreased as spacing between aircraft, whether wake spacing inair (Figure 19) or runway occupancy spacing applied to touchdown (Figure 20), was increased. The trend was non-linear and is presumably dependent on the availability of options for merging and frequency of non-optimal leader/follower weight class pairings, but was not tested in this study.

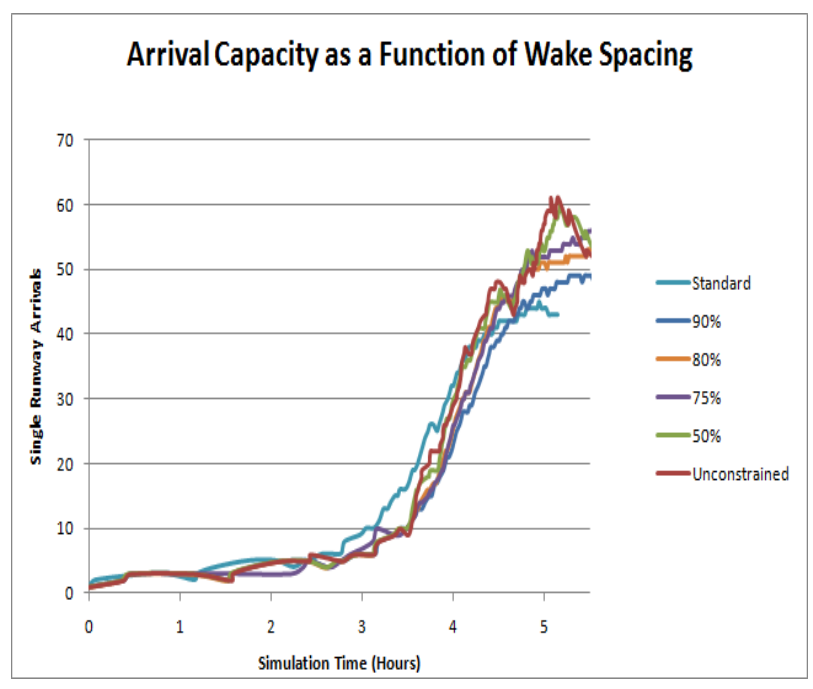

Figure 19. Arrival Capacity Sensitivity, Wake Spacing

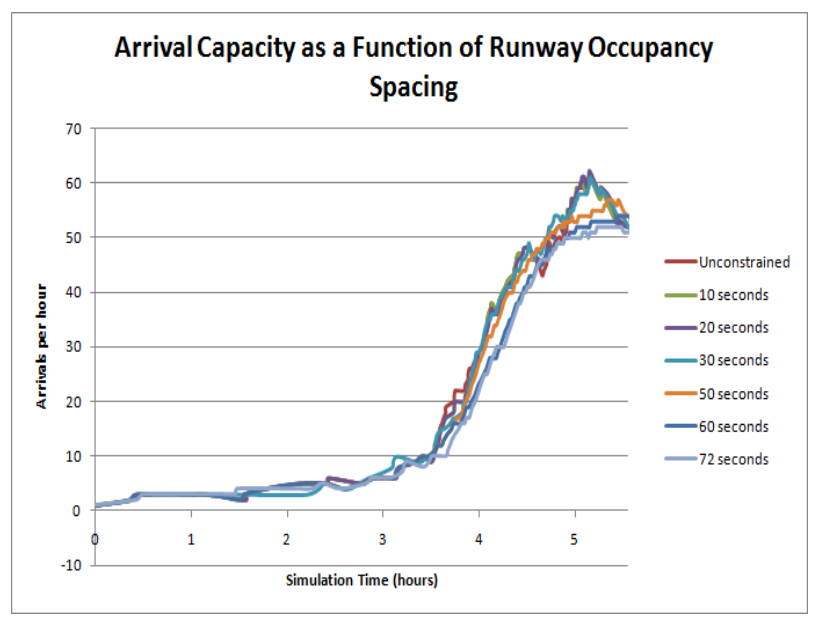

Figure 20. Arrival Capacity Sensitivity, Runway Spacing

\section{Potential Application and Benefit}

The ACES simulation with the M\&S plug-in has potential benefit for both near and far-term research applications. A considerable amount of STAR route development is currently in progress to add Area Navigation (RNAV) and Continuous Descent Approach (CDA) routes to US airports. With additional maturity and validation, the simulation tool could be useful for high-level benefits studies to identify potential risk or payoff in early assessment of new or modified routes. However, near-term application for specific airports or routes would probably require a more detailed validation than the high-level assessment described in this paper. For far-term application, the tool is useful in its current form for comparison of concepts and routing to identify promising technology directions, as needed for project roadmaps. Researchers can use it to take the next step in the investigation of costs and benefits of future concept routes.

\section{Future Work}

Validation and sensitivity testing performed in this study was limited by time constraints to those applications needed for the planned follow-on study of future arrival routing concept benefits. The process of gathering data and answers, however, identified nuances in route modeling that merit further investigation. As concept route configurations for follow-on work are developed, sensitivity testing needed to support those concepts will be performed. These are expected to include the effect on arrival capacity of aircraft mix, of using a prioritization scheme (rather than firstcome-first-served), and of expanded use of aircraft separation by altitude.

Concept routes are currently planned to target near-term, mid-term, and far-term NextGen technology expectations. Near-term concepts will rely on expanded or exclusive use of RNAV and CDA routes. Mid-term concepts will attempt to 
demonstrate possible scenarios to efficiently deal with mixed equipage while incentivizing operators to invest in new technologies. Far-term concepts will represent highly speculative scenarios that maximize available terminal airspace and move completely away from predetermined flight paths or STARs. This work is planned for mid-2011.

\section{References}

1. 2010, Airspace Concepts Evaluation System (ACES) Version 7.1 with M\&S Plug-in, California, NASA Ames Research Center and Intelligent Automation, Inc.

2. Santos, Michel, Arthur Feinberg, Yingchuan Zhang, Yun Teng, Jensan Chen, Nikhil Nigam, Jeremy Smith, 2010, Scheduling the Use of Airborne Merging and Spacing Along Multiple Converging Routes to an Airport, Toronto, AIAA Modeling and Simulation Technologies Conference.

3. 2008, Order 7110.65R, Air Traffic Control, 55-1, Section 10, Washington, D.C., United States Department of Transportation and the Federal Aviation Administration.

4. 2011, Google Earth 4.3.7284.3916 (beta), Google.

5. 2010, AirNav, KATL Hartsfield-Jackson Atlanta International Airport, AirNav LLC, http://www.airnav.com/airport/KATL.

6. 2010, ACES Viewer, Version 3, Ohio, Aerospace Computing, Inc.
7. Shresta,Sanjiv, Dejan Neskovic, Evan Saltzman, Scott Williams, 2008, Terminal Separation Analysis, Measurement, Characterization, and Capacity Estimation MTR080232, McLean, VA, The MITRE Corporation.

8. 2010, FlightAware, Flight Tracker, Arrivals, http://flightaware.com/live/airport/KATL.

\section{Acknowledgements}

The author wishes to thank Gary Lohr, NASA Langley Research Center, for his insight on Air Traffic Control operations. She also wishes to thank Mike Richardson, of the Atlanta TRACON, for his time and insight into the specific challenges and initiatives of traffic flow in the Atlanta region.

2011 Integrated Communications Navigation and Surveillance (ICNS) Conference

May 10-12, 2011 


\section{Appendix A - Atlanta Airport Selected Arrival Procedures}

\section{CANUK Arrival Procedure (obtained from AirNav.com[5])}

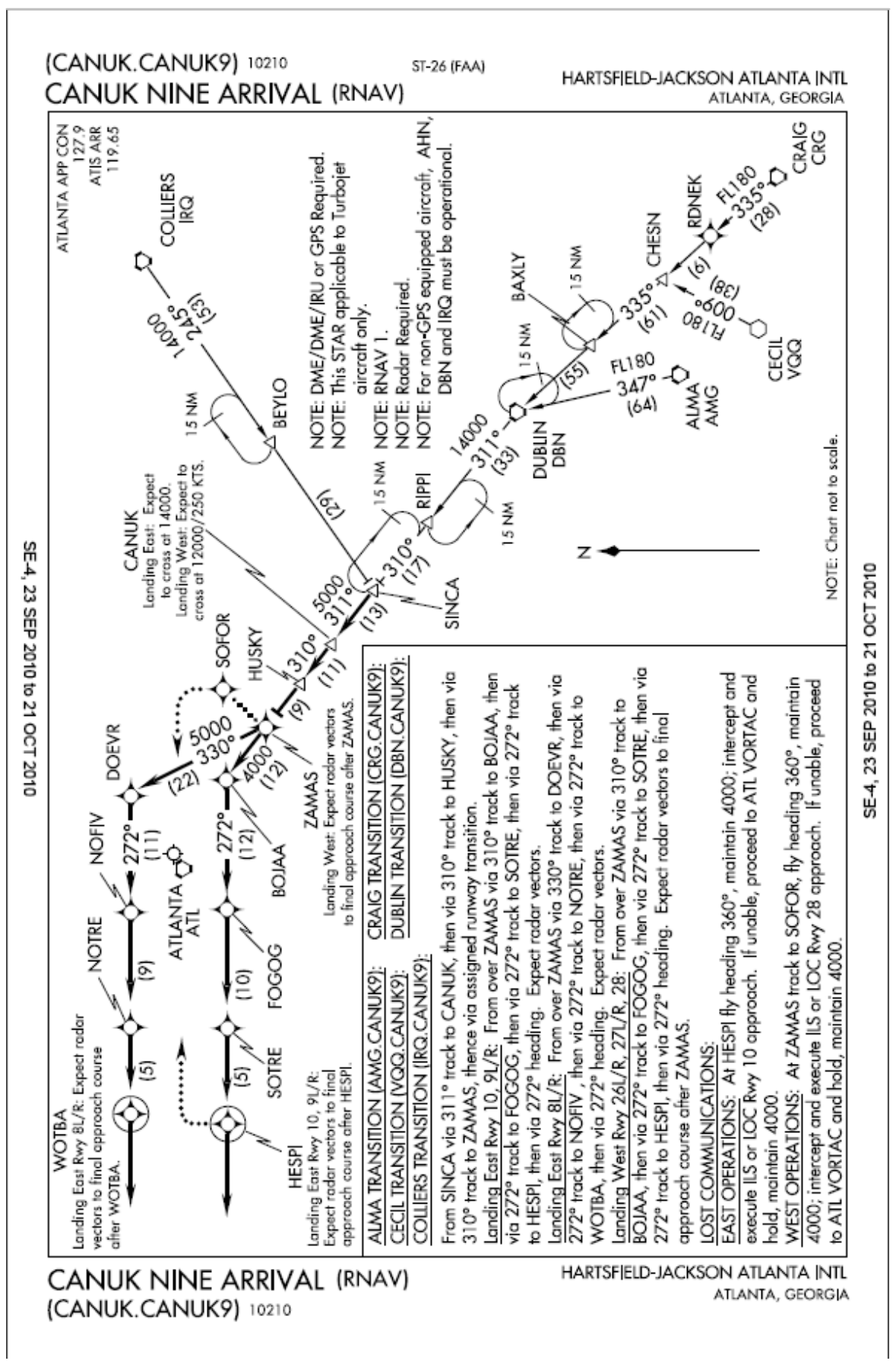




\section{ERLIN Arrival Procedure (obtained from AirNav.com[5])}

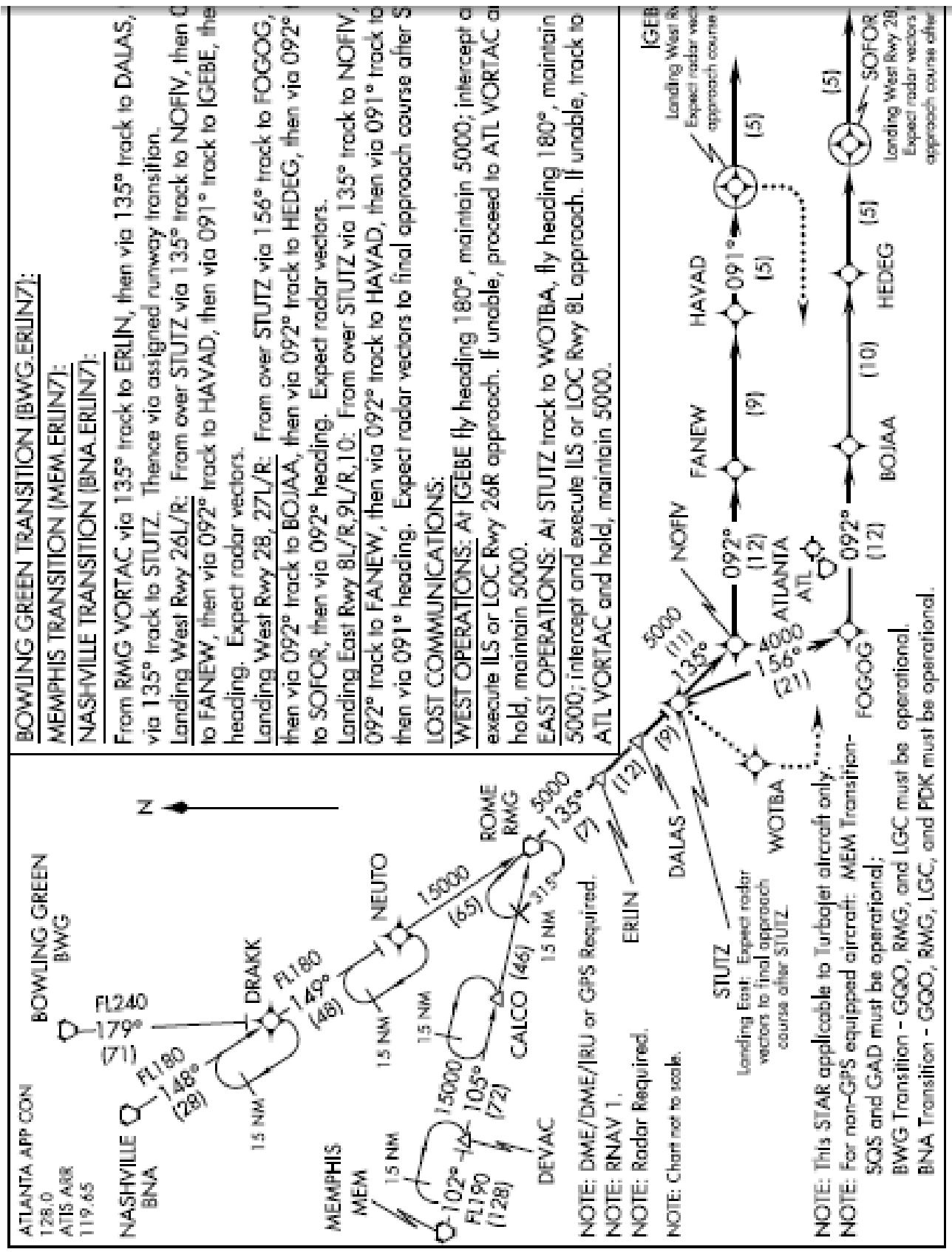

ERLIN SEVEN ARRIVAL (RNAV) (ERLIN.ERLIN7) 10210 


\section{FLCON Arrival Procedure (obtained from AirNav.com[5])}

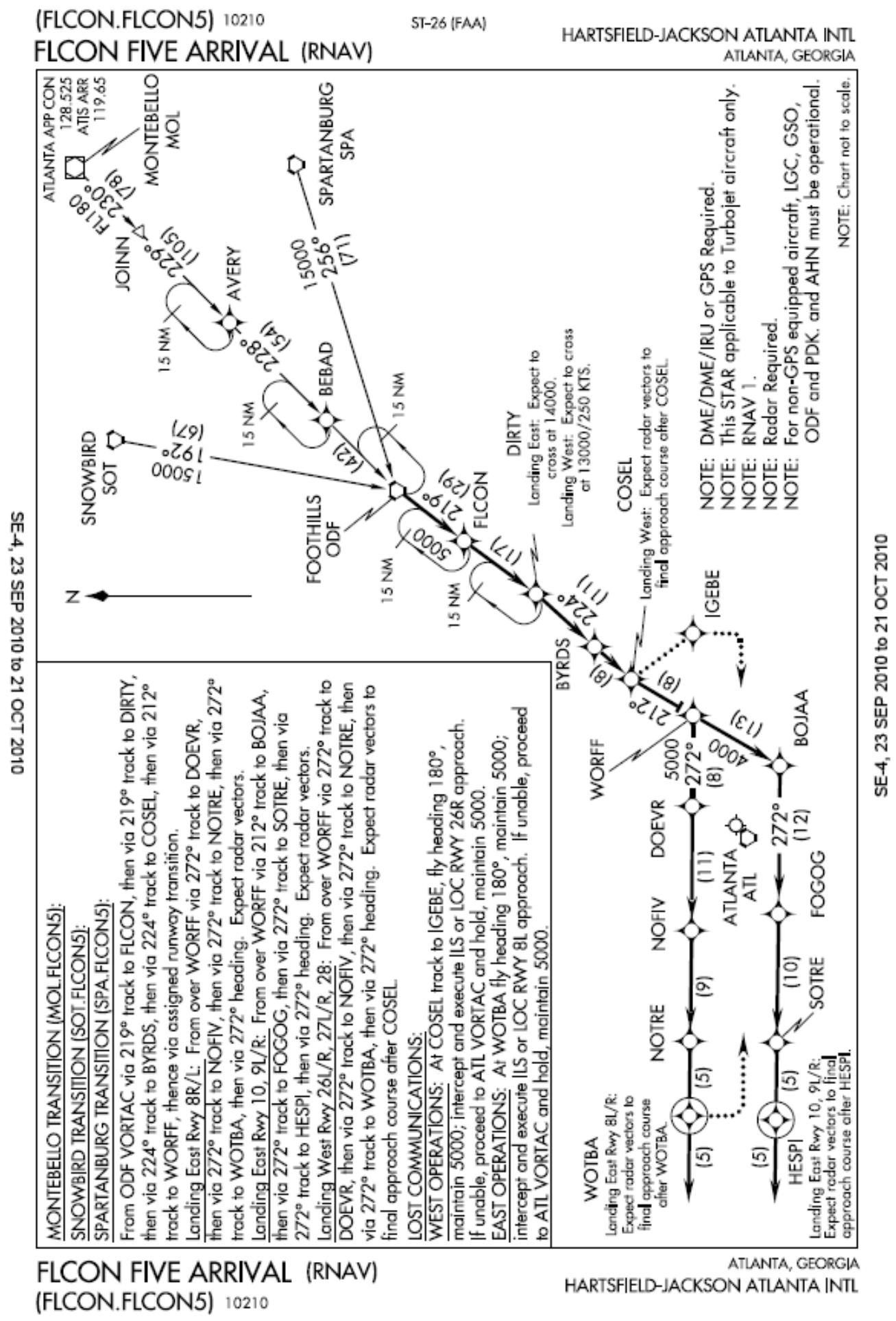




\section{HONIE Arrival Procedure (obtained from AirNav.com[5])}

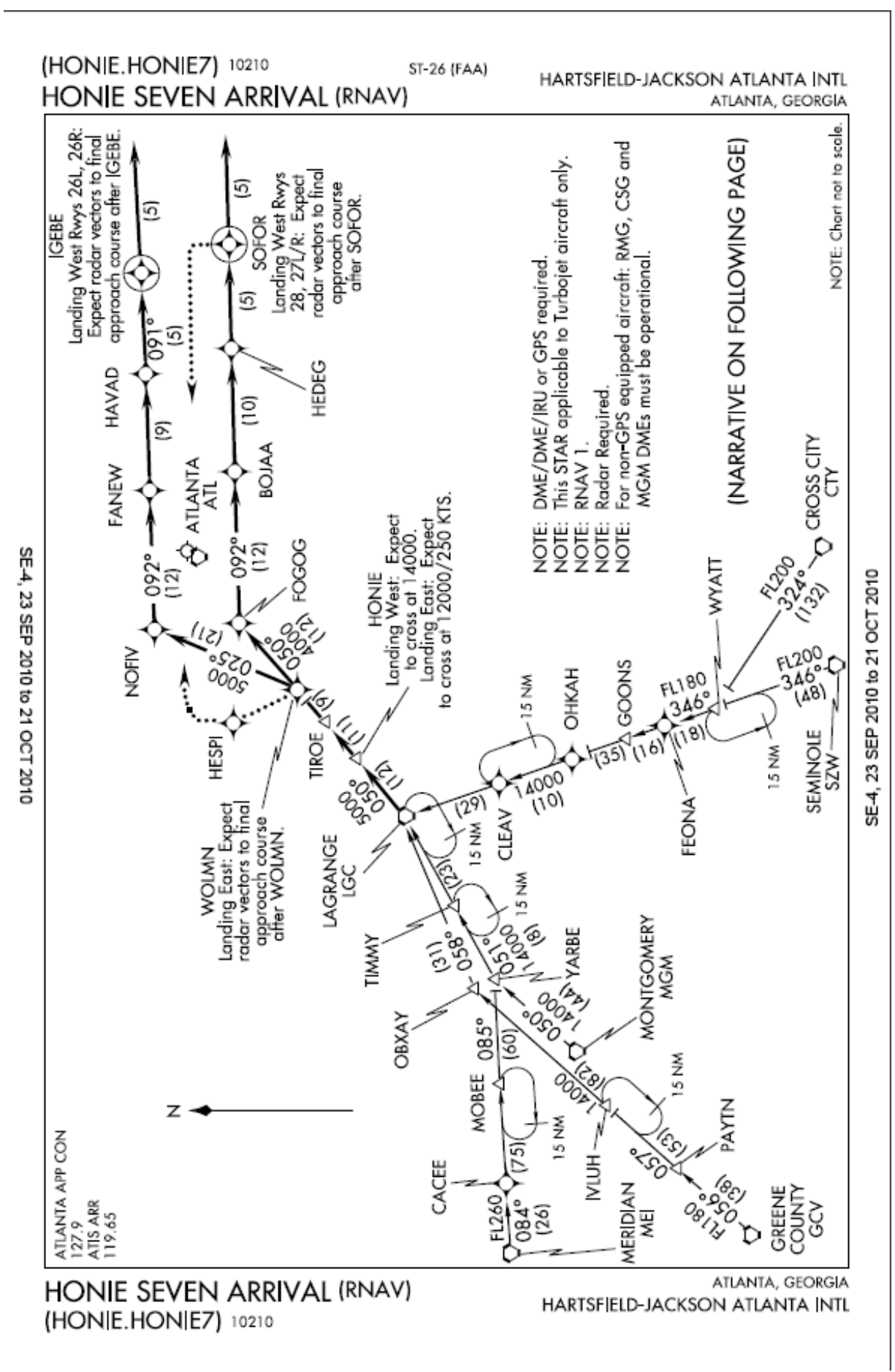


2011 Integrated Communications Navigation and Surveillance (ICNS) Conference

May 10-12, 2011 